\title{
Correction to: Methylthioadenosine promotes remyelination by inducing oligodendrocyte differentiation
}

Beatriz Moreno ${ }^{1,2}$, Gemma Vila', Begoña Fernandez-Diez ${ }^{1}$, Raquel Vázquez ${ }^{1}$, Alessandra di Penta ${ }^{1,3}$, Oihana Errea ${ }^{1}$, Nagore Escala ${ }^{1}$, Andrés Miguez ${ }^{4}$, Jordi Alberch ${ }^{4}$ and Pablo Villoslada ${ }^{1,5,6^{*}}$

\section{Correction to: Multiple Sclerosis and Demyelinating}

Disorders (2017) 2:3.

https://doi.org/10.1186/s40893-017-0020-8

After publication of the article [1], it has been brought to our attention that the full funding acknowledgement is missing from the original article. It should also include the following -

"This work was supported by the Instituto de Salud Carlos III with FEDER funds (Otra forma de hacer Europa) from the European Commission (FIS: PI12/01823)".

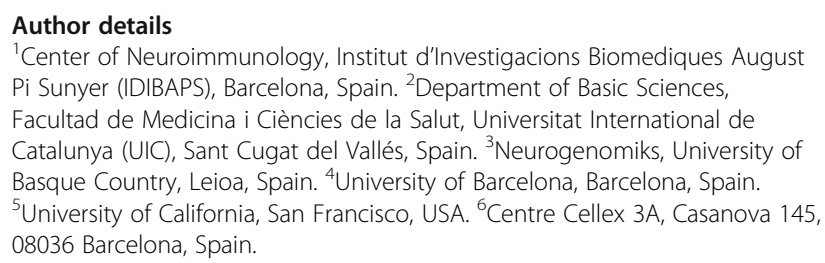

\section{Reference}

1. Moreno B, Vila G, Fernandez-Diez B, Vázquez R, di Penta A, Errea O, et al. Methylthioadenosine promotes remyelination by inducing oligodendrocyte differentiation. Multiple Sclerosis and Demyelinating Disorders. 2017;2(1).

\footnotetext{
* Correspondence: pvilloslada@clinic.ub.es; Pablo.VillosladaDiaz@ucsf.edu ${ }^{1}$ Center of Neuroimmunology, Institut d'Investigacions Biomediques August Pi Sunyer (IDIBAPS), Barcelona, Spain

${ }^{5}$ University of California, San Francisco, USA
} 